\title{
Multifunctional Robotic Guidewire System using Spiral-type Magnetic Microrobot with Magnetic Manipulation
}

\author{
Chang-Ho Yu ${ }^{1}$ and Sung Hoon Kim ${ }^{2 *}$ \\ ${ }^{I}$ Department of Convergence Technology Engineering, Chonbuk National University, 567 Baekje-daero, Jeonju, \\ Jeonbuk 54896, Republic of Korea \\ ${ }^{2}$ Department of Electronics Convergence Engineering, Wonkwang University, 460 Iksandae-ro, Iksan, \\ Jeonbuk 54538, Republic of Korea
}

(Received 21 November 2016, Received in final form 8 December 2016, Accepted 8 December 2016)

\begin{abstract}
This paper presents a new multifunctional active guidewire system for medical applications that uses a magnetic microrobot. The study demonstrated that the proposed microrobot system could swim and be controlled under Low-Reynolds-number $(\mathrm{Re})$ environments in blood vessel models. The prototype of the robotic guidewire, which is driven within a three-axis Helmholtz coil system, consists of a guide-wire, spiral blade, drilling tip, and permanent magnet. The spiral-type microrobot showed stable active locomotion between $3 \mathrm{kA} / \mathrm{m}$ and $9.1 \mathrm{kA} / \mathrm{m}$ under driving frequency up to $70 \mathrm{~Hz}$ in a silicone oil (of viscosity 1000 cst). The microrobot produced a maximum moving velocity of $8.08 \times 10^{-3} \mathrm{~m} / \mathrm{s}$ at $70 \mathrm{~Hz}$ and $9.1 \mathrm{kA} / \mathrm{m}$. In particular, the robotic guidewire produced 3D locomotion with drilling in the three-axis Helmholtz coil system. We verified active locomotion, towing of guidewire, steering, and drilling of the proposed robotic guidewire system through experimental analyses.
\end{abstract}

Keywords : robotic guidewire, magnetic navigation system, spiral-type magnetic microrobot, three-axis helmholtz coil

\section{Introduction}

Multiscale magnetic robots and their magnetic manipulation systems are innovative approaches used for diagnosis and therapy in minimally invasive medicine [13] The greatest advantages of magnetic microrobots are wireless control and battery-free operation inside the human body through the control of external magnetic fields $[4,5]$. In addition, multi-scale fabrication of magnetic robots is possible up to micro/nano scales $[6,7]$. Medical magnetic robots have been applied in targeted drug delivery, drilling in blood vessels, and robotic capsuleendoscopy for diagnosis and therapy [8-11].

One representative application in minimally invasive medicine is an active catheter and guidewire system. Endovascular intervention is a therapeutic minimally invasive approach to treating a cerebral aneurysm from inside the vessel by using various interventional devices (guidewire, catheter, balloon, stent, and so on). A cerebral

(C) The Korean Magnetics Society. All rights reserved.

*Corresponding author: Tel: +82-63-850-6739

Fax: +82-63-850-6739, e-mail: kshoon@wku.ac.kr aneurysm rupture has been one of the most dangerous disorders threatening people's health, and with those current medical facilities, aneurysms are possible to be detected before a rupture. As the process of the treatment of aneurysms involves a high risk that may cause severe damage to the patient, medical doctors are faced with a hard decision of whether to treat aneurysms or not [12].

Conventional catheterization requires a certain human skill because doctors have to control the tip of the catheter or the guide wire by moving it from the outside the patient's body. To overcome these problems, system using active catheters and guidewires has been developed to achieve controllable steering [13]. The tip of active catheter and guidewire system is controlled from outside the body and moves like a snake by utilizing a multi-joint mechanism with distributed shape memory alloy (SMA) actuators $[14,15]$. The catheter can perform several motions, not only bending but also torsional, and can be extended by changing the configuration of the SMA actuators and bias springs. However, such systems are limited in terms of functions.

Typically, magnetic microrobots utilize rolling, rotating, oscillating, and biomimetic mechanisms for active locomo- 
tion within alternating or rotating magnetic fields $[16,17]$. The magnetic spiral-type mechanism utilizes a rotating magnetic field for free-swimming in liquid environments. The applied rotating magnetic field creates a magnetic torque that causes the rotation of the magnetic spiral-type machines. The magnetic torque and the rotation then generate a propulsive force in the environment.

In this study, we proposed a new approach for medical applications based on multifunctional active guidewire system with a magnetic spiral-type microrobot. The study demonstrated that the proposed microrobot system could swim and be controlled under Low-Reynolds-number $(\mathrm{Re})$ environments in blood vessel models. The prototype of the robotic guidewire, which is driven within a threeaxis Helmholtz coil system, consists of a guidewire, spiral blade, drilling tip, and permanent magnet. The proposed robotic guidewire system is capable of active locomotion, steering, towing guidewire, and drilling. Various experimental analyses were performed to verify these functions.

\section{Mechanism and Wireless Magnetic Manipulation}

\subsection{Mechanism of robotic guidewire}

The concept of the proposed guidewire is shown in Fig. 1 (a). The guidewire, which is driven by a uniform rotating magnetic field, includes a spiral-type magnetic microrobot with a drill tip for active locomotion and drilling blood clots in blood vessels, respectively. The magnetic microrobot consists of a cylindrical $\mathrm{NdFeB}$

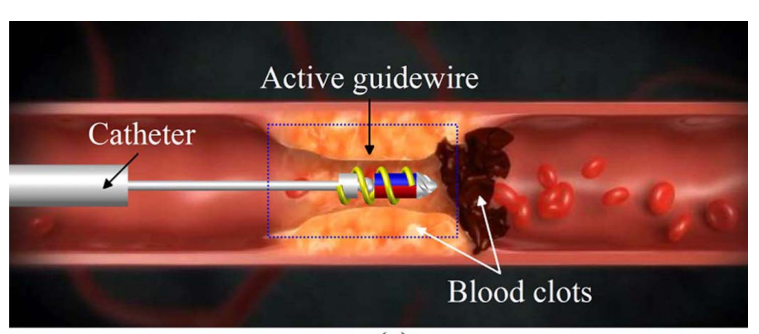

(a)
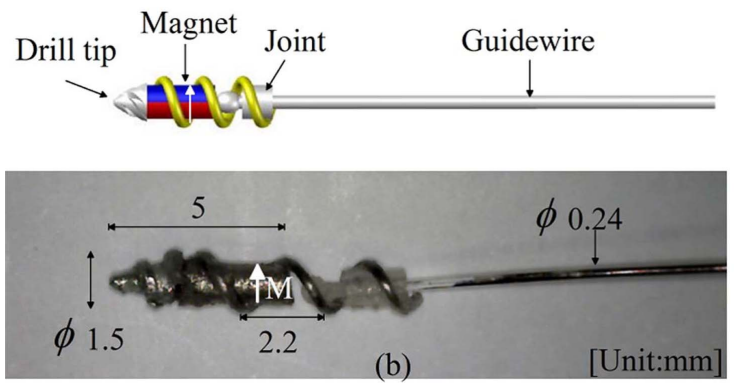

Fig. 1. (Color online) (a) Concept of the proposed robotic guidewire system and (b) the proposed mechanism and prototype of the robotic guidewire. permanent magnet (vector product of magnetic moment M is $1.1 \times 10^{-3} \mathrm{Am}^{2}$, BHmax is approximately $310 \mathrm{~kJ} / \mathrm{m}^{3}$, and coercive force $\mathrm{Hcb}$ and $\mathrm{Hcj}$ are approximately 880 $\mathrm{kA} / \mathrm{m}$ and $960 \mathrm{kA} / \mathrm{m}$, respectively), a tungsten wire $(\Phi$ $0.24 \mathrm{~mm}$ ) for a spiral blade, an Fe-based drilling tip, and a joint for connecting the guidewire, as shown in Fig. 1 (b). The mechanism of the spiral blade is the right-handed screw. The blade angle and pitch are $50^{\circ}$ and $2.2 \mathrm{~mm}$, respectively. The direction of the magnetic moment in the robot is the radial direction. Therefore, the microrobot generates rotational motion within the rotating magnetic field. Thus, the spiral-type magnetic microrobot creates a propulsive force that facilitates active locomotion in various fluid environments. The joint connects the microrobot and the guidewire and prevents the rotation of the guidewire.

\subsection{Wireless magnetic manipulation of robotic guide-} wire

The proposed guidewire system generates active locomotion of the spiral-type magnetic microrobot within the rotating magnetic field. Figure 2 (a) shows a magnetic manipulation system based on three-axis Helmholtz coils. The control system generates a uniform rotating magnetic field. Because of the uniform magnetic field, the proposed robotic guidewire is driven by a magnetic torque. Therefore, the end-effector of the guidewire system is synchronized by the applied rotating magnetic field.

The plane of the rotating magnetic field was controlled
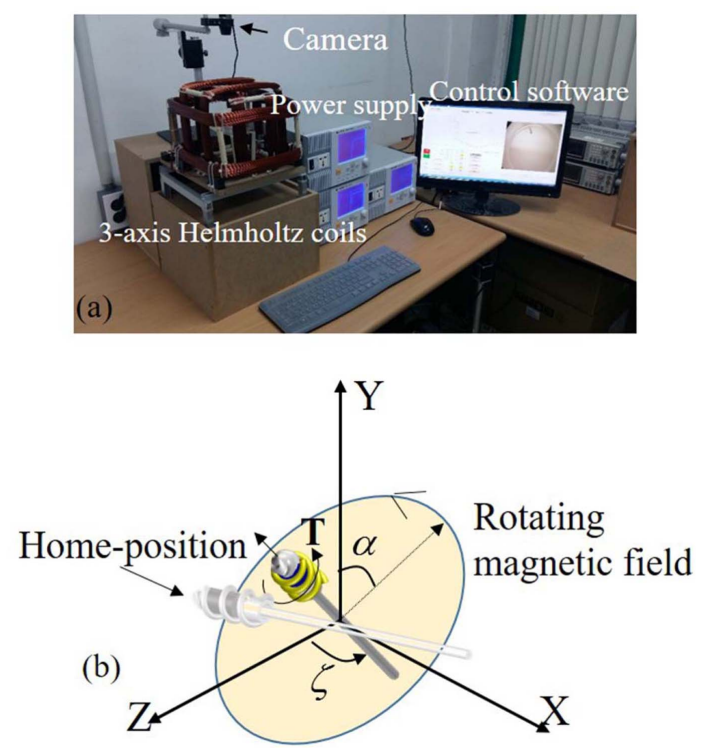

Fig. 2. (Color online) (a) Wireless magnetic manipulation system based on three-axis Helmholtz coils, (b) Steering method using the three-axis Helmholtz coils: $\alpha$ and $\zeta$ represent the zenith and azimuth angles of the plane of the magnetic field. 
through control of the two angles of $\alpha$ and $\zeta$ for realizing three-dimensional steering function, as shown in Fig. 2 (b). The two angles determine the moving direction within the three-axis Helmholtz coils because of changes in the direction of magnetic torque.

The applied rotating magnetic field is expressed by the sum of the vectors of the magnetic fields from three-axis Helmholtz coils. The applied current signals for controlling active locomotion with steering are expressed as follows:

$$
I=\left(\begin{array}{c}
I_{X} \\
I_{Y} \\
I_{Z}
\end{array}\right)=I_{m}\left(\begin{array}{c}
\cos \zeta \sin \alpha \sin \omega t-\sin \zeta \cos \omega t \\
\cos \zeta \cos \omega t+\sin \zeta \sin \alpha \sin \omega t \\
\cos \alpha \sin \omega t
\end{array}\right)
$$

where $I_{x}, I_{y}$, and $I_{x}$ denote the input current of the Helmholtz coils current signals. $I_{m}$ is the maximum input current. Figure 3 shows the calculated current signal according to changes in the steering angles of $\alpha$ and $\zeta$ at $1 \mathrm{~Hz}$. The initial plane of the rotating magnetic field is $\mathrm{YZ}$ plane, and $I_{y}$ and $I_{z}$ signals were activated, whereas $\alpha=45^{\circ}$ and $\zeta=45^{\circ}$ generated $I_{y}, I_{z}$, and $I_{x}$. Therefore, the plane of the rotating magnetic field is changed from the $\mathrm{YZ}$ plane to the XYZ plane. Because of the input current signals, the magnetic fields of a three-axis Helmholtz coils are written as follows:

$$
\mathbf{H}_{x, y, z}=\left(\begin{array}{l}
H_{x} \\
H_{y} \\
H_{z}
\end{array}\right)=H_{0}\left(\begin{array}{c}
\cos \zeta \sin \alpha \sin \omega t-\sin \zeta \cos \omega t \\
\cos \zeta \cos \omega t+\sin \zeta \sin \alpha \sin \omega t \\
\cos \alpha \sin \omega t
\end{array}\right)
$$

where $H_{0}$ is the field strength: $H_{0}=n I_{m} R^{2} /\left(R^{2}+x^{2}\right)^{3 / 2}, n$ is the number of coil turns, and $R$ and $x$ are the radius of the Helmholtz coil and the distance between one pair of Helmholtz coil, respectively. The magnitude of the rotating magnetic field can be expressed as follows:

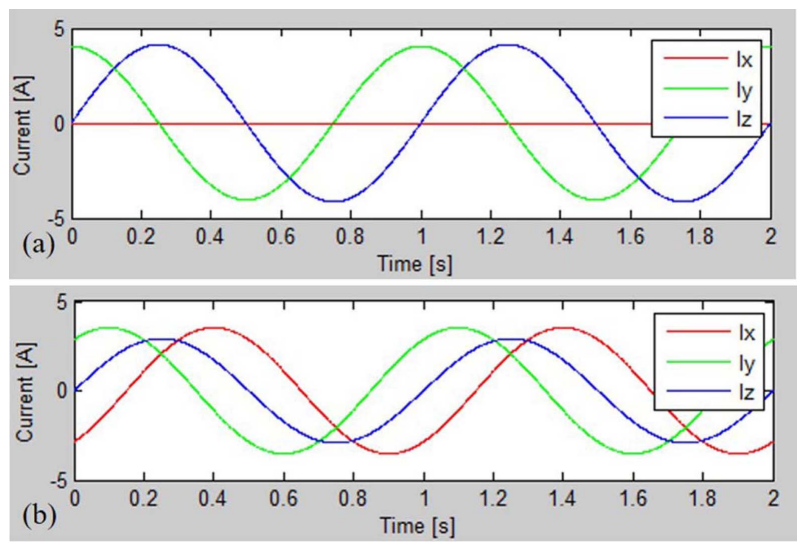

Fig. 3. (Color online) (a) Calculated current signal for 4.579 $\mathrm{kA} / \mathrm{m}$ at $\alpha=0^{\circ}$ and $\zeta=0$ and (b) Current signals at $\alpha=45^{\circ}$ and $\zeta=45$.

$$
\left|\mathbf{H}_{r o t}\right|=\sqrt{\begin{array}{l}
\sin ^{2} \omega t\left(\sin ^{2} \zeta \sin ^{2} \alpha+H_{0}^{2} \cos ^{2} \alpha\right. \\
\left.+H_{0}^{2} \cos ^{2} \zeta \sin ^{2} \alpha\right)+\cos ^{2} \omega t\left(H_{0}^{2} \cos ^{2} \zeta+\sin ^{2} \zeta\right)
\end{array}}
$$

Figure 2 (b) shows a magnetic moment vector $\mathbf{m}$ rotating in the YZ plane at the home position: $\mathbf{m}=\left(0, \boldsymbol{m}_{0} \cos \theta\right.$, $\boldsymbol{m}_{0} \sin \theta$ ) Therefore, the driving torque of the robot for steering within the manipulating system is expressed as follows (See Fig. 2) [18]:

$$
\begin{aligned}
& \mathbf{T}=\mu_{0} \mathbf{m} \times \mathbf{H}_{x, y, z} \\
& \mathbf{T}=\mu_{0} m_{0} H_{0}\left\{\begin{array}{l}
(\cos \alpha \cos \theta \sin \omega t-\sin \theta(\cos \zeta \cos \omega t \\
+\sin \zeta \sin \alpha \sin \omega t)) \mathbf{i} \\
-\sin \theta(\sin \zeta \cos \omega t-\cos \zeta \sin \alpha \sin \omega t) \mathbf{j} \\
+\cos \theta(\sin \zeta \cos \omega t-\cos \zeta \sin \alpha \sin \omega t) \mathbf{k}
\end{array}\right\}
\end{aligned}
$$

where $\mu_{0}$ denotes the permeability of free space. When the applied rotating magnetic field is clockwise, the endeffector of the guidewire generates forward locomotion, whereas counter-clockwise magnetic field causes backward locomotion because of the right-handed screw mechanism on the robot body. When the rotating magnetic field is on plane YZ, the robotic guidewire produces thrust force toward $\mathrm{X}$-axis by the spiral-type blade. The thrust force $\left(\mathrm{F}_{\mathrm{t}}\right)$ of the robot is expressed as follows:

$$
\mathbf{F}_{\mathbf{t}}=\mathbf{F}_{\mathbf{n}} \cos \beta_{\mathbf{n}} \cos \delta-\mathbf{F}_{\mathbf{f}} \sin \delta
$$

where $F_{f}$ is the friction force opposing the movement on the blade surface: $\mathbf{F}_{\mathbf{f}}=\mu_{\mathbf{s}} \cdot \mathbf{F}_{\mathbf{n}}, \mathbf{F}_{\mathbf{n}}$ is the reactive force $\left(\mathbf{F}_{\mathbf{n}}=\mathbf{F}_{\mathbf{t}} /\left(\cos \beta_{\mathbf{n}} \cos \delta-\mu_{\mathrm{s}} \sin \delta\right)\right), \mu_{\mathrm{s}}$ is the friction coefficient, and $\beta_{\mathrm{n}}, \delta$ are the blade angle and the lead angle, respectively, as shown in Fig. 4.

\section{Experimental Analysis}

To verify the proposed mechanism, various experimental analyses using the magnetic manipulating system were conducted. Figure 5 shows the variations in movement velocity according to the changes in the driving frequency up to $100 \mathrm{~Hz}$ at the applied rotating magnetic field of

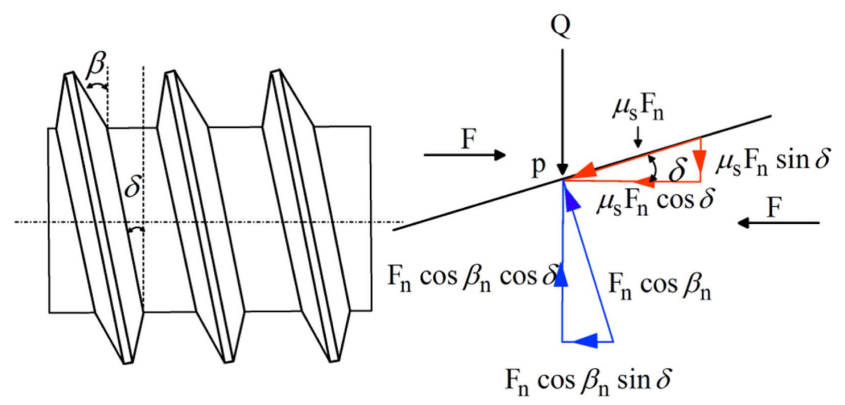

Fig. 4. (Color online) Force analysis on a screw mechanism. 


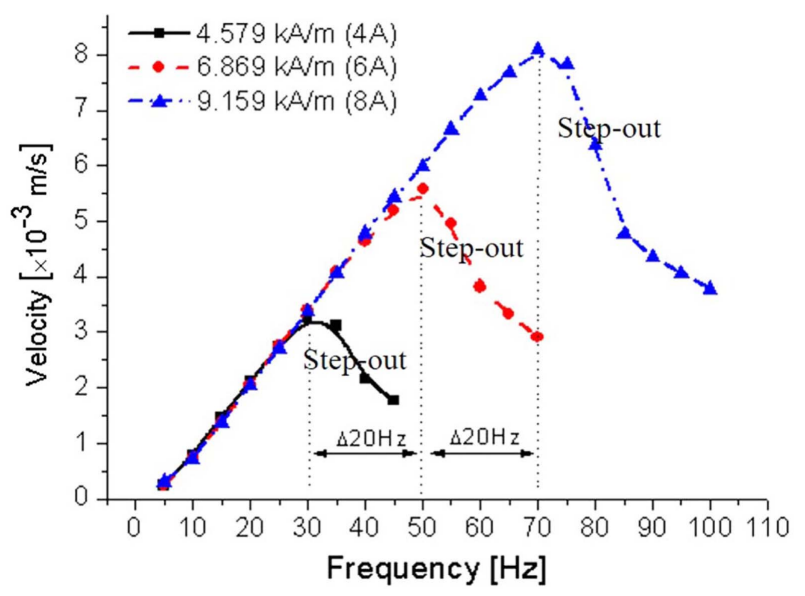

Fig. 5. (Color online) Results of moving velocity according to changes in driving frequency at the applied rotating magnetic fields of $4.579,6.689$, and $9.159 \mathrm{kA} / \mathrm{m}$, respectively.

$4.579,6.869$, and $9.159 \mathrm{kA} / \mathrm{m}$ in a silicone oil (of viscosity $1000 \mathrm{cst}$ ).

The applied fields of $4.579,6.869$, and $9.159 \mathrm{kA} / \mathrm{m}$ produced maximum velocities of $3.26 \times 10^{-3}, 5.58 \times 10^{-3}$ and $8.08 \times 10^{-3} \mathrm{~m} / \mathrm{s}$ at the driving frequency of 30,50 , and $70 \mathrm{~Hz}$, respectively. The driving frequencies of 30,50 and $70 \mathrm{~Hz}$ become maximum frequencies for linearity at each magnetic field. If the viscosity is changed, maximum frequencies are changed at the equivalent magnetic fields. Through the results, stable operating ranges were observed. When the driving frequencies exceeded the maximum points, movement velocities were dramatically decreased by decreasing driving torque because the exceeded maximum frequencies caused a decrease in the angle between the magnetic moment and the applied magnetic field. The driving frequency at which the robot reaches its maximum velocity is called the step-out frequency. In this range, the velocity decreases dramatically, while the frequency increases. The step-out ranges are different according to the input frequency and magnetic field, because of the different magnetic torque.

For an active guidewire, the most important function is steering with active locomotion. Figure 6 shows the steering angle of the robotic guidewire. The initial position of the plane of the rotating magnetic field is plane YZ, as shown in Fig. 6 (a). The plane of the rotating magnetic field was changed from plane YZ to plane YZX according to the changes in angle $\zeta$, that is $30^{\circ}, 60^{\circ}$, and $90^{\circ}$ at magnetic fields of $4.579,5.724,6.869$, and $8.014 \mathrm{kA} / \mathrm{m}$ respectively, as shown in Fig. 6 (b). In the results, the stronger magnetic field was found to cause a higher steering angle. When angle $\zeta$ was $30^{\circ}$ at 4.579 and $6.869 \mathrm{kA} /$ $\mathrm{m}$, actual steering angles were $20.24^{\circ}$ and $21.84^{\circ}$, respec-

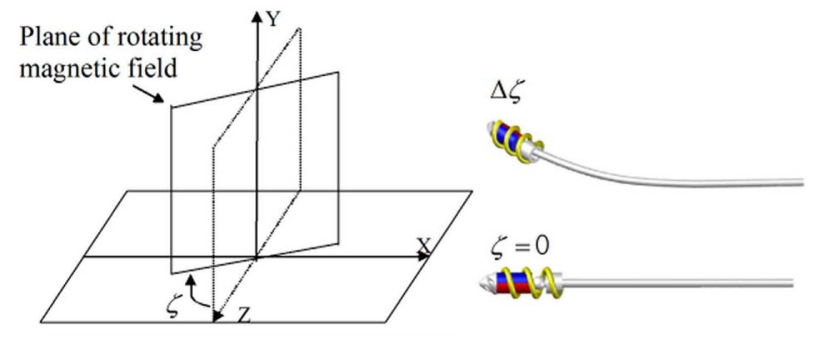

(a)

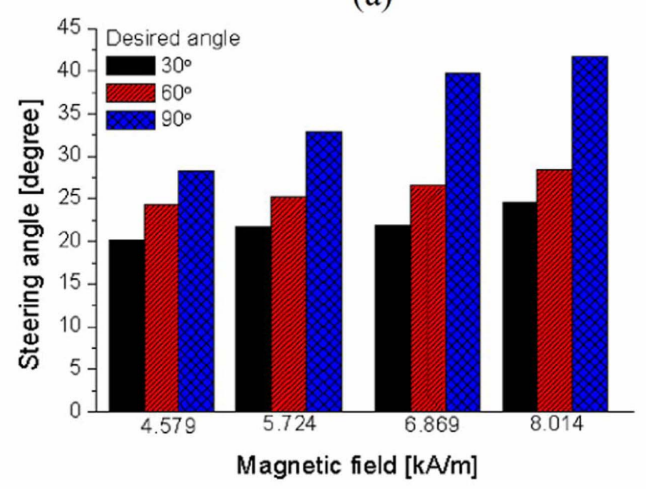

(b)

Fig. 6. (Color online) (a) Experimental method for steering in the magnetic manipulating system. $\zeta$ denotes the rotational angle of plane of rotating magnetic field. (b) Results of steering angles in the magnetic manipulating system according to changes in the applied magnetic fields with angle $\zeta=30^{\circ}$, $60^{\circ}$, and $90^{\circ}$, and, respectively.

tively.

In this case, steering accuracy achieved was 67.5 and $72.8 \%$, respectively, when angle $\zeta$ was $60^{\circ}$ at 4.579 and $6.869 \mathrm{kA} / \mathrm{m}$, the steering angle of the robotic guidewire became $24.32^{\circ}$ and $26.54^{\circ}$, respectively. Under these conditions, steering accuracy was decreased up to 40 and 44.2 $\%$, respectively. Angle $\zeta=90^{\circ}$ resulted in the highest steering angle, whereas the steering accuracy was the lowest: steering angle was $41.79^{\circ}$ and accuracy was 46.43 $\%$ at $8.014 \mathrm{kA} / \mathrm{m}$. The reason of the results is the tension of the guidewire. These results show that a gradual rate of change of angle $\zeta$ is required to achieve higher precision steering.

Figure 7 shows in-vitro tests of active locomotion, steering, and drilling abilities of the proposed robotic guidewire system. First, active locomotion with steering was verified, as shown in Fig. 7 (a). For active locomotion, a magnetic field of $4.579 \mathrm{kA} / \mathrm{m}$ and driving frequency of $8 \mathrm{~Hz}$ was applied when angle $\zeta=0^{\circ}$ (plane YZ). Bifurcation geometry mock-up of the artificial blood vessel, which is made from 3D print using a blood vessel model, has a diameter of $2.5 \mathrm{~mm}$ and bifurcation angle of $50^{\circ}$. To reach the steering angle of $50^{\circ}$, angle $\zeta$ was gradually increased at an interval of $10^{\circ}$. Figure 7 (b) shows active 

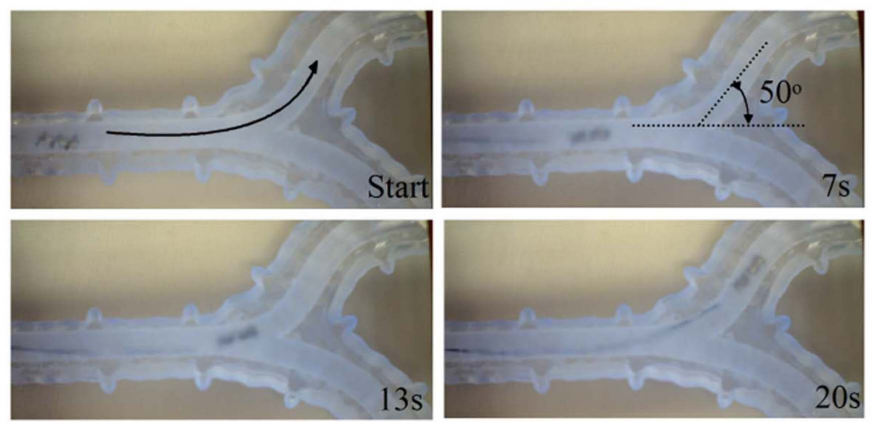

(a)
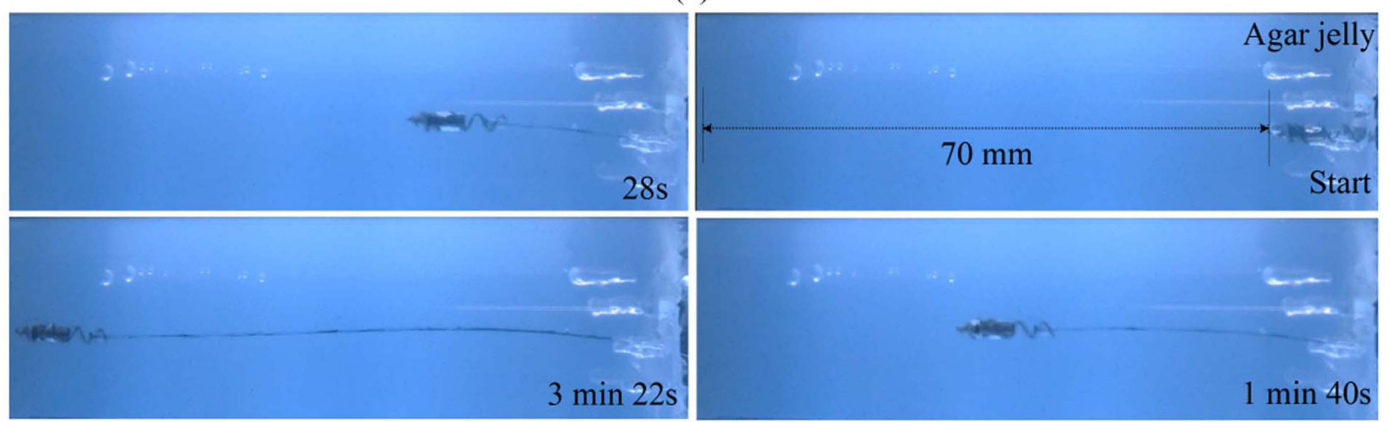

(b)

Fig. 7. (Color online) Experimental results of active locomotion-based steering and drilling: (a) active locomotion with steering at the applied magnetic field of $4.579 \mathrm{kA} / \mathrm{m}$ and $8 \mathrm{~Hz}$ : steering pathway has an angle of $50^{\circ}$, (b) active locomotion (tow of guidewire) and drilling in an agar jelly: driving frequency is $10 \mathrm{~Hz}$ at $4.579 \mathrm{kA} / \mathrm{m}$.

locomotion with drilling function in agar jelly. To fabricate the agar jelly, $1 \mathrm{~g}$ of agar powder was mixed with $200 \mathrm{ml}$ of water. For drilling in the agar jelly, magnetic field of $4.579 \mathrm{kA} / \mathrm{m}$ and $10 \mathrm{~Hz}$ was applied. Under these conditions, movement velocity of the robotic guidewire was $0.34 \times 10^{-6} \mathrm{~m} / \mathrm{s}$. At the equivalent driving conditions, the robotic guidewire showed movement velocity of $0.8 \times 10^{-3} \mathrm{~m} / \mathrm{s}$ in a silicone oil. When the robotic guidewire conducted drilling with active locomotion, the driving frequencies of 10 and $20 \mathrm{~Hz}$ created thrust force of approximately $6.5 \times 10^{-3}$ and $14.5 \times 10^{-3} \mathrm{~N}$, respectively, at the applied field of $4.579 \mathrm{kA} / \mathrm{m}$ and $10 \mathrm{~Hz}$.

\section{Discussion and Conclusion}

In this study, a robotic guidewire system based on the spiral-type magnetic microrobot was proposed. The robotic guidewire, which is controlled by an external rotating magnetic field, performed active locomotion, steering, towing of guidewire, and drilling. To verify these functions, various experiments were performed in a silicone oil and agar jelly. Because the robotic guidewire is synchronized by the applied rotating magnetic field, movement velocity depends on the driving frequency. In addition, driving magnetic fields determine the linear ranges of driving frequency. Steering is the most important func- tion of an active guidewire. As regards precision steering of the robotic guidewire, a rapidly changing angle of plane of the magnetic field caused higher steering error. That is a low rate of change of plane of the magnetic field results in high-precision steering. These results were verified through in vitro study. The previously reported most active guide-wires only conduct steering without active locomotion, whereas the proposed guide-wire shows steering, active locomotion, and drilling. In particular, the end-effector (microrobot) was able to fabricate under a diameter of $1.5 \mathrm{~mm}$.

\section{Acknowledgments}

This research was supported by Wonkwang University in 2015 .

\section{References}

[1] J. J. Abbott, K. E. Peyer, M. C. Lagomarsino, L. Zhang, L. X. Dong, I. K. Kaliakatsos, and B. J. Nelson, Int. J. Robot. Res. 28, 1434 (2009).

[2] A. Ghosh and P. Fischer, Nano Lett. 9, 2243 (2009).

[3] B. J. Nelson, I. K. Kaliakatsos, and J. J. Abbott, Annual Review of Biomedical Engineering 12, 55 (2010).

[4] K. Ishiyama, M. Sendoh, and K. I. Arai, JMMM. 242, 41 (2002). 
[5] S. H. Kim, S. Hashi, and K. Ishiyama, J. Appl. Phys. 109, 07E318 (2011).

[6] L. Zhang, J. Abbott, L. Dong, K. D. Bell, and B. J. Nelson, Appl. Phys. Lett. 94, 064017 (2009).

[7] F. Qiu and B. J. Nelson, Engineering 1, 21 (2015).

[8] S. Jeon, G. Jang, H. Choi, S. Park, and J. Park, IEEE Trans. Magn. 47, 2403 (2011).

[9] S. H. Kim and K. Ishiyama, IEEE Trans. Mechatronics 19, 1651 (2013).

[10] S. Pane, O. Ergeneman, K. M. Sivaraman, T. Luhmann, H. Hall, and B. J. Nelson, Proc. IEEE Int' Conf. Nano/ Molecular Medicine and Engineering 148 (2010).

[11] S. Yim and M. Sitti, IEEE Trans. Robotics 28, 183 (2012).

[12] Y. H. Kim, X. Xu, and J. S. Lee, Annals of Biomedical Engineering 38, 2274 (2010).
[13] S. M. Jeon and G. H. Jang, IEEE Trans. Magn. 48, 4062 (2012).

[14] G. Lim, K. Park, M. Sugihara, K. Minami, and M. Esashi, Sensors Actuators A 56, 113 (1999).

[15] A. Sakes, E. Regar, J. Dankelman, and P. Breedveld, Cardiovascular Engineering and Technology 7, 103 (2016).

[16] S. Aramaki, S. Kaneko, K. Arai, Y. Takahashi, H. Adachi, and K. Yanagisawa, Proceedings of IEEE Sixth International Symposium on Micro Machine and Human Science 115 (1995).

[17] S. Sudo, S. Segawa, and T. Honda, Journal of Intelligent Material Systems and Structure 17, 729 (2006).

[18] S. H. Kim, S. Hashi, and K. Ishiyama, IEEE Trans. Magn. 49, 3488 (2013). 